\title{
A GraphML-based Visualization Framework for Workflow-Performers' Closeness Centrality Measurements
}

\author{
Min-Joon Kim ${ }^{1 *}$, Hyun Ahn², Minjae Park ${ }^{3}$ \\ ${ }^{1}$ Department of School of Integrated Technology, YONSEI UNIVERSITY \\ Incheon, 406-840, South Korea \\ [e-mail: minjoon@yonsei.ac.kr] \\ ${ }^{2}$ Department of Computer Science, KYONGGI UNIVERSITY \\ Suwon, 443-760, South Korea \\ [e-mail: hahn@kgu.ac.kr] \\ ${ }^{3}$ Engineering Innovation Department, R\&D Center, BISTel, INC. \\ Seoul, 137-891, South Korea \\ [e-mail: mjpark@bistel-inc.com] \\ *Corresponding Author: Minjae Park
}

Received April 7, 2015; revised June 12, 2015; accepted July 8, 2015;

published August 31, 2015

\begin{abstract}
A hot-issued research topic in the workflow intelligence arena is the emerging topic of "workflow-supported organizational social networks." These specialized social networks have been proposed to primarily represent the process-driven work-sharing and work-collaborating relationships among the workflow-performers fulfilling a series of workflow-related operations in a workflow-supported organization. We can discover those organizational social networks, and visualize its analysis results as organizational knowledge. In this paper, we are particularly interested in how to visualize the degrees of closeness centralities among workflow-performers by proposing a graphical representation schema based on the Graph Markup Language, which is named to ccWSSN-GraphML. Additionally, we expatiate on the functional expansion of the closeness centralization formulas so as for the visualization framework to handle a group of workflow procedures (or a workflow package) with organizational workflow-performers.
\end{abstract}

Keywords: workflow-supported organizational social network (WSSN), information control net (ICN), closeness centralization measures/vector (CCV), workflow model, graph markup language (graphML), organizational knowledge management, workflow intelligence

A preliminary version of this paper was presented at ICONI 2014, and was selected as an outstanding paper.

This research was supported by the MSIP(Ministry of Science, ICT and Future Planning), Korea, under the "IT Consilience Creative Program" (NIPA-2014-H0201-14-1002) supervised by the NIPA(National IT Industry Promotion Agency). Also, it was partially supported by the GRRC of Gyeonggi Province. 


\section{Introduction}

In recent, the workflow literature has been interested in the concept of workflow intelligence being supported by the integration of workflow modeling techniques [1], organizational intelligence techniques [3,4], and social network analysis techniques [5]. The concept starts from the strong belief that social relationships and collaborative behaviors among workflow-supported people affect the overall performance as well as the degrees of working productivity in performing the real business operations under the control of workflow-supported organizations [6]. As a consequence, pioneering research and development activities $[7,8,9,10,11,12]$ on workflow-supported people (so-called workflow-performers) have been delivered into the literature. In particular, the authors' research group has initiated the human-centered workflow intelligence as organizational knowledge.

As a means of visualizing human-centered workflow knowledge, this paper proposes a visualization framework focusing on measuring the centralizations of workflow performers, which is one of the key practice indicators to identify the important or prominent performers within a workflow procedure, and can be analyzed by the typical social network analysis technique, centrality. The most widely used centrality measurement techniques are degree ${ }^{1}$, closeness $^{2}$, between-ness ${ }^{3}$, and eigenvalue. These measures vary in their applicability to non-directed and directed relations, and differ at the individual performer and the group performers of the partial or complete workflow procedure levels. In particular, we confine the scope of the measurements to the closeness centralization measurement for individual and group workflow-performers within a workflow procedure. The closeness centralization measurement reflects how near a workflow-performer is to the other workflow performers in enacting a workflow procedure, and through which we can numerically measure and quantify the degree of work-intimacy and collaboration of each workflow performer. It implies how quickly a workflow-performer can interact with others by directly communicating or through very few intermediaries. Therefore, in this paper our eventual goal is to formalize a visualization framework that extensively applies to quantify and visualize the degrees of work-intimacy and collaboration of all performers involved in a workflow model, a workflow package (a group of inter-relevant workflow models), or a group of workflow packages on a workflow-supported organization.

In terms of the composition of the paper, we survey the related works of this research first. In the next consecutive sections, we expatiate the detailed formalisms of the proposed framework consisting of analysis phase and visualization phase, in company with an operational example, and finalize the practical implications of the closeness centralization measurement technique and its graphML-based graphical representations in a workflow-supported organization.

\footnotetext{
${ }^{1}$ The degree centrality measures the extent to which a workflow-performer connects to all others.

${ }^{2}$ The closeness centrality measures how near a workflow-performer is to the others.

${ }^{3}$ The betweenness centrality measures how other workflow-performers control or mediate the relations between dyads that are not directly connected.
} 


\section{Related Works}

Recently, the workflow literature has just dug out an emerging research issue, which is the human-centered workflow intelligence coping with social and collaborative structures on workflow-supported organizations. Traditionally, the management literature has been discussing about the relationships between knowledge management and organizational performance, and they eventually found out a series of meaningful results $[15,16,17]$ proving the fact that there exists a considerable degree of semantic linking between human-centered structural knowledge and organizational culture and performance. There are two approaches to visualize the semantic linking. One is to use the traditional statistical analysis techniques [3], the other is to employ the sophisticated social network analysis techniques already proved in the social science domain and summarily introduced in [5] and [19]. The authors' research group has employed these sophisticated social network analysis techniques, such as centrality, prestige, and clique techniques, to visualize the human-centered workflow intelligence. In particular, we have been actively adopting the centrality technique in analyzing the workflow-supported org-social networking knowledge, so far. The centrality technique is subdivided into degree-centrality [13], closeness-centrality [12, 20], betweenness-centrality [21], and eigenvalue-centrality so as to be elaborately applied into a real organizational world.

Until now, almost all research and development activities are mainly concerned about how to discover and analyze the workflow-supported organizational social networking knowledge, whereas the literature is rarely interested in how to effectively, efficiently, and even beautifully visualize the discovered or/and analyzed knowledge. The only one emphasizing the visualization of the knowledge and its analyzed results was [22], in which the authors developed a display function to visualize the degree centralization measurements of the performers engaged in enacting a workflow model. However, because the display function doesn't adopt any graphical toolkits or libraries $[23,24,25]$ to visualize the graphical objects, it always needs a certain amount of additional manual-operations, such as re-positioning, resizing, or re-arranging operations, to gracefully and properly beautify the graphical objects making up the displayed knowledge. Therefore, as an upgraded version of [22], this paper proposes a visualization approach to dramatically improve the quality of beautification as well as the quality of efficiency in visualizing the closeness centralization measures. The visualization approach devised a schema formatted in the extended XML-based graph markup language [14], ccGraphML, so as to graphically express the closeness centrality vector by adopting the well-known information visualization toolkits [24] like Prefuse, JFreeChart, and $\log 4 j$.

\section{A GraphML-based Visualization Framework}

In this section, we formulate a visualization framework for measuring the closeness centralizations that enable us to quantify the levels of work-intimacy and collaboration among workflow-performers. The framework is a kind of procedural framework that starts from a GraphML-based schema of workflow-supported organizational social networks and goes on to a graphical representation of closeness centrality measures, as illustrated in Fig. 1.

In the next consecutive subsections, we describe the details of the functional transformations of the visualization framework through a series of formulas and algorithms, and their operational examples. 


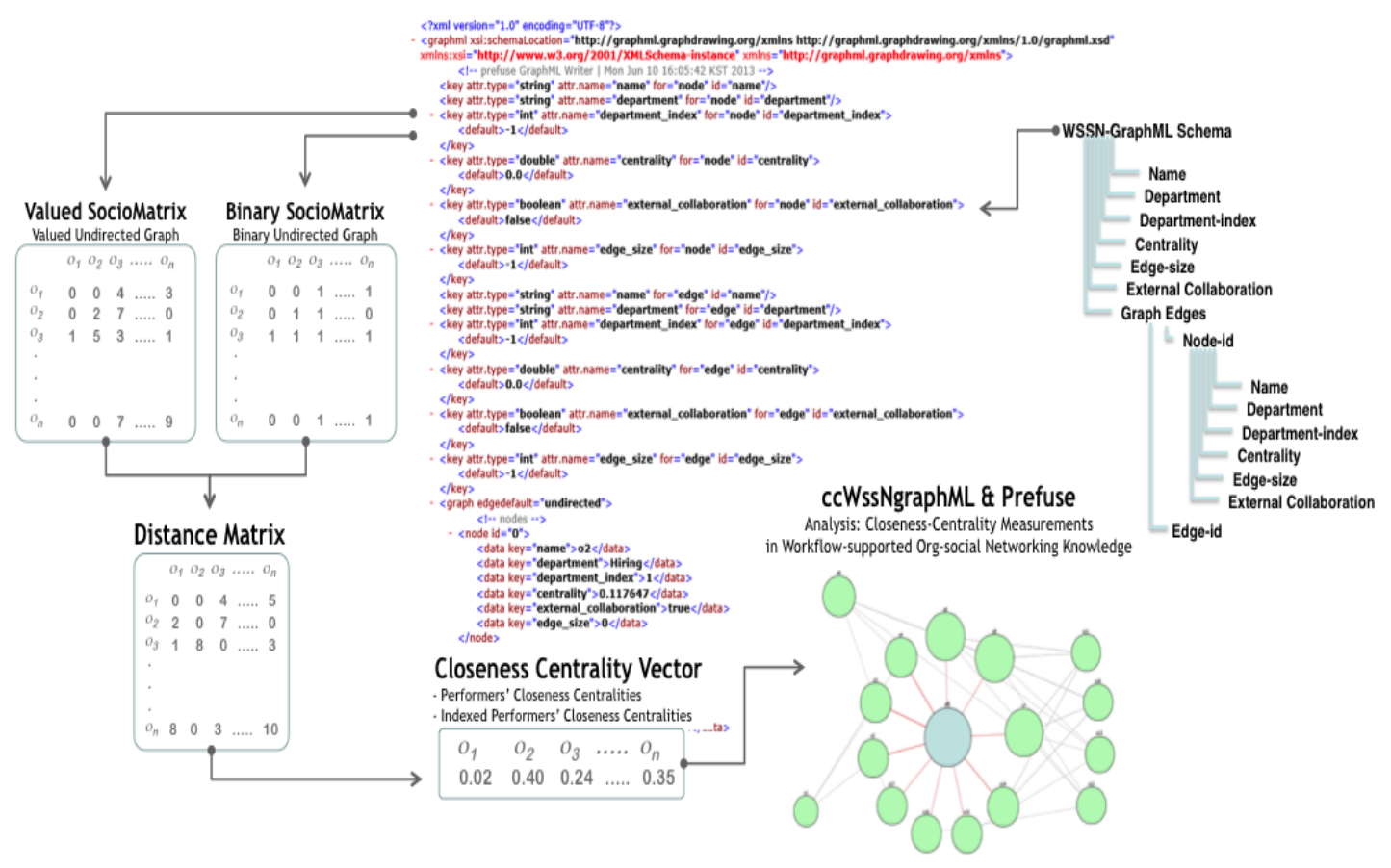

Fig. 1. A GraphML-based Visulization Framework

\subsection{An XML Schema for GraphML-based WSSNs}

The purpose of this subsection is to define an XML schema for GraphML-based workflow-supported organizational social networks that are abbreviated to WSSNs. The activity-performer associations consisting of the activity-role associations and the role-performer associations in the information control net of a workflow model form a graph of the corresponding WSSN by transitively applying these two associations, as followings:

- The activity-role association: for any activity $(\alpha),\left\{\alpha \mid \varepsilon_{p}(\alpha)=\left\{\eta_{i}\right\}^{\wedge} \eta_{i} \in \mathbf{P}\right\}$, where $\mathbf{P}$ is the set of roles, $\left\{\eta_{1}, \eta_{2}, \ldots, \eta_{n}\right\}$, means that the activity $\alpha$ is associated with a single role out of $\mathbf{P}$; also, for any role $(\eta),\left\{\eta \mid \varepsilon_{a}(\eta)=\left\{\alpha_{s}, \ldots, \alpha_{m}\right\}^{\wedge}\left\{\alpha_{s}, \ldots, \alpha_{m}\right\} \subset \mathbf{A}\right\}$, where $\mathbf{A}$ is the set of activities, $\left\{\alpha_{1}, \alpha_{2}, \ldots, \alpha_{m}\right\}$, means that the role $\eta$ is associated with one or more activities out of A. Summarily, activity-to-role association is one-to-one relationship, whereas role-to-activity association is one-to-many relationship.

- The role-performer association: for any role $(\eta),\left\{\eta \mid \pi_{c}(\eta)=\left\{\emptyset_{s}, \ldots, \emptyset_{k}\right\}^{\wedge}\left\{\emptyset_{s}, \ldots, \varnothing_{k}\right\} \subset \mathbf{C}\right\}$, where $\mathbf{C}$ is the set of performers, $\left\{\emptyset_{1}, \emptyset_{2}, \ldots, \emptyset_{n}\right\}$, means that the role $\eta$ is associated with one or more performers out of $\mathbf{C}$; also, for any performer(ø), $\left\{\varnothing \mid \pi_{p}(\varnothing)=\left\{\eta_{s}, \ldots, \eta_{m}\right\} \wedge\right.$ $\left.\left\{\eta_{s}, \ldots, \eta_{m}\right\} \subset \mathbf{P}\right\}$, where $\mathbf{P}$ is the set of roles, means that the performer $\varnothing$ is associated with one or more roles out of $\mathbf{P}$. Summarily, both role-to-performer association and performer-to-role association are many-to-many relationships.

- Based upon these two types of associations, we are able to transitively obtain the activity-to-performer associations from an information control net, and we know that both the activity-to-performer association and performer-to-activity association are many-to-many relationships. Conclusively speaking, not only more than one performers 
can participate in enacting an activity, but also a performer is able to participate in enacting one or more activities.

[Definition 1] Workflow-Supported Organizational Social Network Model. The workflow-supported organizational social network model is formally defined as $\boldsymbol{\Lambda}=(\sigma, \psi, \mathrm{S}$, E), over a set $\mathbf{C}$ of performers, and a set $\mathbf{A}$ of activities, where

- $\mathrm{S}$ is a finite set of coordinators or coordinator-groups connected from some external workflow-supported org-social network models;

- E is a finite set of coordinators or coordinator-groups connected to some external workflow-supported org-social network models;

- $\sigma=\sigma_{i} \cup \sigma_{o} / *$ Control-Precedence Relationships */

where, $\sigma_{o}: \mathbf{C} \rightarrow \wp(\mathbf{C})$ is a multi-valued function mapping a performer to its sets of (immediate) candidate-successors, and $\sigma_{i}: \mathbf{C} \rightarrow \wp(\mathbf{C})$ is a multi-valued function mapping a performer to its sets of (immediate) candidate-predecessors;

- $\psi=\psi_{i} \cup \psi_{o} / *$ Activity-Acquisition Relationships */

where, $\psi_{i}: \mathbf{C} \rightarrow \wp(\mathbf{C})$ is a multi-valued function returning a bag ${ }^{4}$ of previously worked activities, $(\mathbf{K} \subseteq \mathbf{A})$, on directed $\operatorname{arcs},\left(\sigma_{i}(\varnothing), \varnothing\right), \varnothing \in \mathbf{C}$, from $\sigma_{i}(\varnothing)$ to $\varnothing$; and $\psi_{o}$ : $\mathbf{C} \rightarrow \wp(\mathbf{C})$ is a multi-valued function returning a set of acquisition activities, $(\mathbf{K} \subseteq$ A), on directed arcs, $\left(\varnothing, \sigma_{o}(\varnothing)\right), \varnothing \in \mathbf{C}$ from $\varnothing$ to $\sigma_{o}(\varnothing)$;

The WSSN graph is formally represented by the workflow-supported organizational social network model, as given in the formal definition, [Definition 1]. The behaviors of the model are revealed through incoming and outgoing directed arcs labeled as activities between a pair of associated performers. The directed arcs manifest two kinds of behaviors - control-precedence and activity-acquisition - between the associated performers, through which we are able to obtain the work-precedence (candidate-predecessor knowledge/candidate-successor knowledge) knowledge and the activity-acquisition knowledge among performers in a workflow procedure. In terms of defining the performer's predecessors and successors, we would use the prepositional word, "candidate," because a role-performer association is a one-to-many mapping relationship. Additionally, the performer selecting and binding mechanism has to choose one out of the assigned performers of the role during a corresponding workflow instance's execution time.

In principle, the workflow-supported organizational social network model is graphically represented by a directed graph characterized by some combinations of multiple-incoming arcs, multiple-outgoing arcs, cyclic, self-transitive, and multiple-activity associations on arcs, and which needs to be transformed to an undirected graph. For measuring the closeness centralities among the associated performers, the WSSN graph, which is a directed graph, needs to be transformed into an undirected graph, too.

\footnotetext{
${ }^{4}$ The bag theory is same to the set theory except allowing duplicated members.
} 


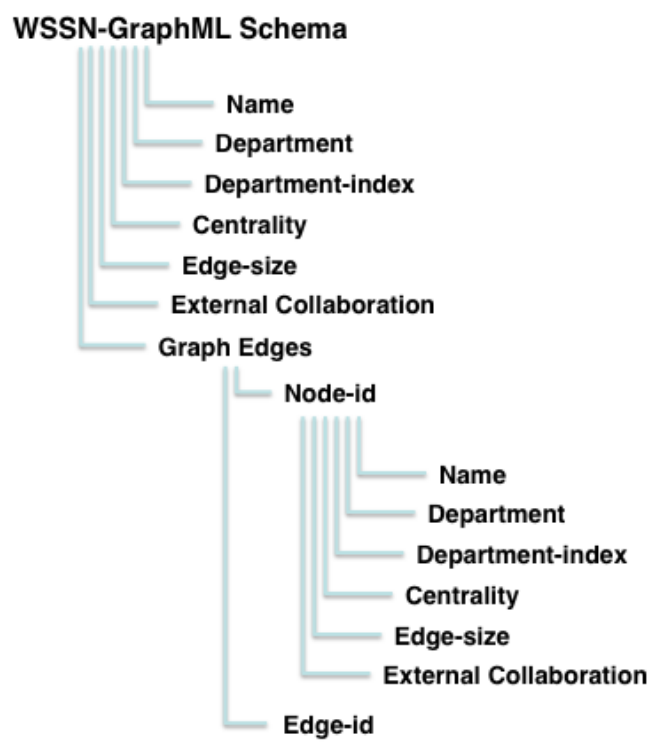

Fig. 2. A GraphML-based WSSN Schema

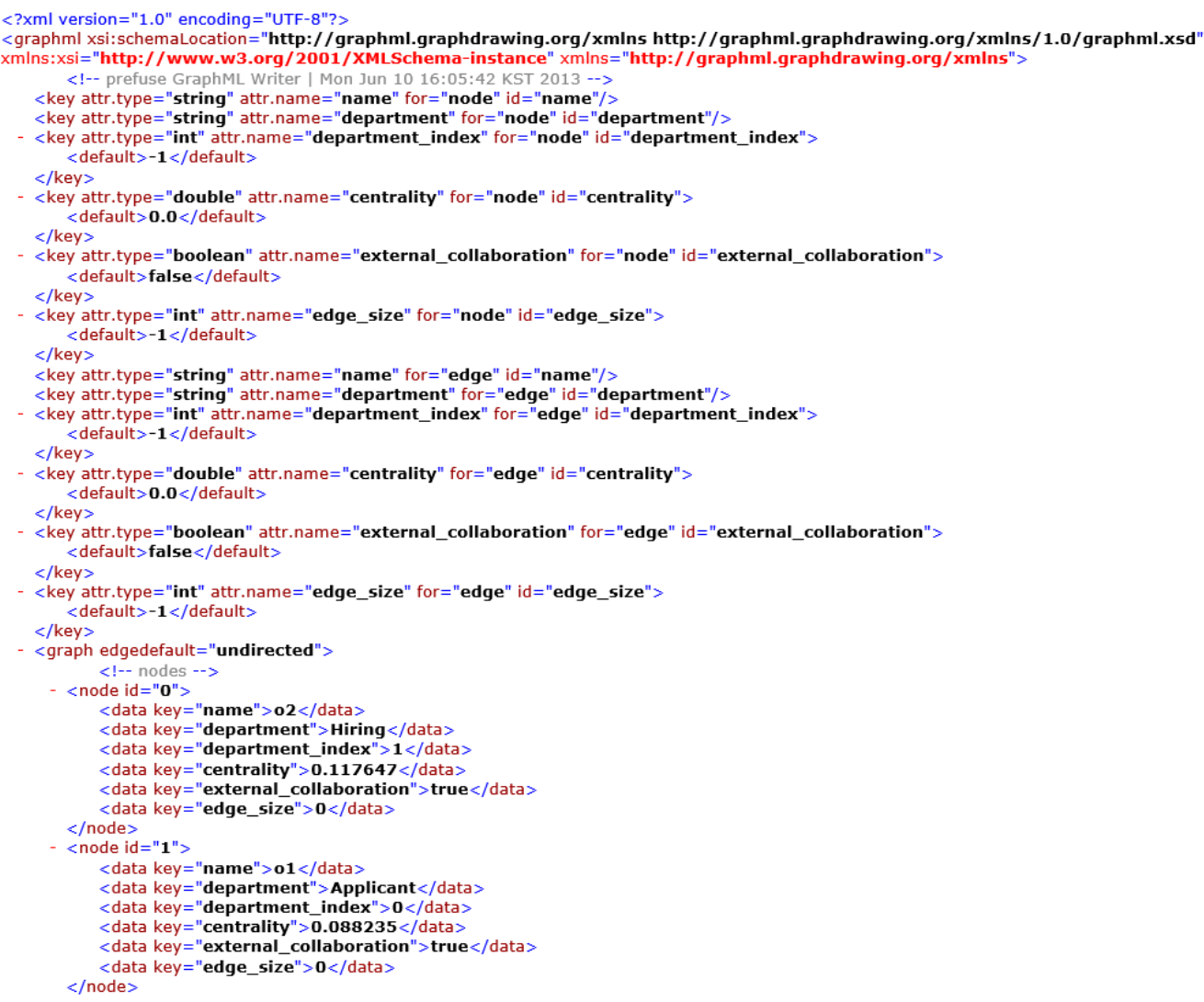

Fig. 3. An XML Snapshot of an Examplified GraphML-based WSSN

In order to format WSSNs in an XML-based textual form, we devise an XML schema of WSSNs, and named it as the WSSN-GraphML, which is based on the well-known XML-based graphical markup language, GraphML. As depicted in Fig. 2, the XML schema of the 
proposed WSSN-GraphML is able to basically express the graphical magnitude and color of the individual node for each workflow-performer. Originally, the graph markup language, GraphML, is an XML-based graph definition language comprising nodes and edges. The nodes and edges are represented by the key attributes defined in the tags of $\langle$ Node Id $>$ and $<$ Edge Id>, respectively. Fig. 3 shows an XML snapshot of an exemplified WSSN-GraphML-based WSSN model.

Moreover, each node can be extended by appending any additional properties, such as node-color, node-size, and others. Also, the graph itself can be characterized by graph-type attribute as either undirected graph or directed graph. In order to sophisticatedly describe the workflow-supported organizational social network by using GraphML, we can supplement the domain-specific attributes to <Node Id $>$ and $<$ Edge Id $>$. Especially, to graphically reflect the strengths of the relationships (or work-intimacies) between workflow performers, we can add the attribute of Weight to <Edge Id>.

\subsection{Mathematical Representations for GraphML-based WSSNs}

\section{Binary Directed SocioMatrix Transformation Algorithm:}

Input A workflow-supported org-social network, $\boldsymbol{\Lambda}=(\sigma, \psi, \mathbf{S}, \mathbf{E})$;

Output Two symmetric binary SocioMatrices, $\mathbf{Z}_{\text {in }}^{\mathrm{b}}[\mathrm{N}, \mathrm{N}]$ and $\mathbf{Z}_{\text {out }}^{\mathrm{b}}[\mathrm{N}, \mathrm{N}]$, where $\mathrm{N}$ is the number elements in the set of $\mathbf{C}$ actors.

\section{Begin Procedure \\ Initialize all entries of $\mathbf{Z}_{\text {in }}^{\mathrm{b}}[\mathrm{N}, \mathrm{N}]$ To Zeroes; \\ Initialize all entries of $\mathbf{Z}_{\text {out }}^{\mathrm{b}}[\mathrm{N}, \mathrm{N}]$ To Zeroes;}

For $(\forall \varnothing \in \mathbf{C})$ Do

Begin

/* The Incoming Relations of $\mathbf{Z}^{\mathrm{b}}$ in $[\mathrm{N}, \mathrm{N}] * /$

Set One To entries of $\mathbf{Z}^{\mathrm{b}}{ }_{\text {in }}\left[\varnothing\right.$, each member of $\left.\sigma_{i}(\varnothing)\right]$;

/* The Outgoing Relations of $\mathbf{Z}_{\text {out }}^{\mathrm{b}}[\mathrm{N}, \mathrm{N}] * /$

End

Set One To entries of $\mathbf{Z}_{\text {out }}^{\mathrm{b}}\left[\varnothing\right.$, each member of $\left.\sigma_{o}(\varnothing)\right]$;

\section{End Procedure}

The WSSN graph is a formal representation of the workflow-supported org-social network model, and its mathematical representation is expressed in terms of SocioMatrix [5] introduced in the social network literature. In order to mathematically measure the closeness centralizations, the WSSN graph needs to be transformed in SocioMatrix by a WSSN-to-SocioMatrix transformation algorithm. SocioMatrix can be refined on two groups of subtle matrices - binary directed/undirected SocioMatrix and valued directed/undirected SocioMatrix. We use to construct a sociogram [5] that is a two-dimensional diagram for depicting the precedence relationships among performers. The term, directed, indicates the directed-precedence relationships or ties from one at the tail to another at the arrowhead, whereas the term, undirected (no arrowheads), implies mutual-precedence relationships. Likewise, when a directed/undirected WSSN graph is transformed to SocioMatrix, the term, binary, implies the most basic measurement, presence or absence of a tie, which is a dichotomy indicated by the binary value of 1 or 0 , respectively; also SocioMatrix may include valued cells, reflecting the intensity of relationships or ties, such as frequency, tie strength, or magnitude of associations, and therefore the cell entries in SocioMatrix can vary from 0 to the maximum level of dyadic interactions. 


\subsection{Closeness Centrality Analysis of the GraphML-based WSSNs}

In this paper, we focus on quantitatively measuring the degree of closeness centralization by borrowing the well-known formulas [5] in the social network analysis literature. The analysis phase carries out two functional calculations. One is to calculate the geodesic distances among performers, and the other is to measure the individual closeness centralizations by using the result (the geodesic distances) of the former calculation. The ultimate goal of the analysis phase aims to answer to the following essential question:

- How quickly can a performer interact with others in enacting the associated workflow procedure by communicating directly or through very few intermediaries?

That is, through the closeness centrality concept and its measurements we can obtain a reasonable level of analysis results, which is enough to answer to the above question as well as the other questions stated in the beginning of the paper. The closeness centrality measures can be applied to the individual performer (individual closeness centrality) as well as the group of performers (group closeness centrality).

\subsubsection{The Individual Closeness Centrality}

Based upon the SocioMatrices, $\mathbf{Z}_{\text {in }}^{\mathrm{b}}[\mathrm{N}, \mathrm{N}], \mathbf{Z}_{\text {out }}^{\mathrm{b}}[\mathrm{N}, \mathrm{N}], \mathbf{Z}^{\mathrm{b}}[\mathrm{N}, \mathrm{N}], \mathbf{Z}_{\text {in }}^{\mathrm{v}}[\mathrm{N}, \mathrm{N}], \mathbf{Z}_{\text {out }}^{\mathrm{v}}[\mathrm{N}, \mathrm{N}]$, and $\mathbf{Z}^{\mathrm{v}}[\mathrm{N}, \mathrm{N}]$, we are able to calculate the closeness centrality measures by applying the formula given in (1) [5].

- The Index of Individual Closeness Centrality

$$
\operatorname{Cc}\left(\phi_{i}\right)=\frac{1}{\sum_{j=1}^{N} d\left(\phi_{i}, \phi_{j}\right)}(i \neq j)
$$

Formula (1) is for measuring an individual performer's closeness centrality. The term, $d\left(\emptyset_{i}\right.$, $\left.\emptyset_{j}\right)$, in the denominator is a function of geodesic distance that is the length of the shortest path out of all reachable paths from $\emptyset_{i}$ to $\emptyset_{j}$. The conceptual implication of the individual closeness centrality refers to how quickly a performer can interact with others by communicating directly or through very few intermediaries. Conclusively, from one of the SocioMatrices with $\mathrm{N}$ performers, the index of individual closeness centrality is computed as the inverse of the sum of the geodesic distances between performer $\emptyset_{i}$ and the $(\mathrm{N}-1)$ other performers. It is also charged with the function of geodesic distances, $d\left(\emptyset_{i}, \emptyset_{j}\right)$, for all workflow-performers by iteratively applying the function for $\mathrm{N}$ times as many workflow-performers. Eventually, we are able to measure not only the standardized index of individual closeness centrality, but also the index of group closeness centrality for a workflow-supported org-social network.

\subsubsection{The Closeness Centrality Vectors}

Based on the geodesic distance matrix, DistanceMatrix $[\mathrm{N}, \mathrm{N}]$, that is transformed from the previous function, we can measure the closeness centralizations of all the individuals. The following formula (2) is for carrying out the closeness centrality vectors' calculations. The result of the formula is the closeness centrality vector, $\left[\mathrm{Cc}\left(\varnothing_{1}\right), \ldots, \mathrm{Cc}\left(\varnothing_{n}\right)\right]$. Each quantity of the vector is computed as the inverse of the sum of the geodesic distances between its corresponding performer, $\emptyset_{i}$, and the $(\mathrm{N}-1)$ other performers. As you see, the measured indices computed from formula (2) can never be 0.0 , because division by zero is mathematically undefined. Thus, the index of individual closeness centrality cannot be computed for an isolated performer, which is the case of that only a single performer is 
assigned to enacting all activities of the corresponding workflow procedure. Also, we can predict that the lowest index, which is the case of the highest sum of the geodesic distances between a focal performer and others, comes out from a performer either in a relatively large network or in a small network with relatively long geodesic distances from others.

- The Closeness Centrality Vector

$$
\left[C c\left(\phi_{i}\right)\right]_{i=1}^{N}=\left[\frac{1}{\sum_{j=1}^{N} \text { DistanceMatrix }\left[\phi_{i}, \phi_{j}\right]}(i \neq j)\right]_{i=1}^{N}
$$

- The Standardized Closeness Centrality Vector

$$
\left[C_{C}^{S}\left(\phi_{i}\right)\right]_{i=1}^{N}=\left[(N-1) \cdot C c\left(\phi_{i}\right)\right]_{i=1}^{N}
$$

Formula (3) is for standardizing the index of individual closeness centrality by multiplying by $(N-1)$, in which the corresponding performer is excluded from the total number of performers. Suppose that an individual performer has the closest distance to all others, which means that the performer has a direct tie to everyone in the network. Then, the computed values of indexes will be various according to their network sizes. In order to control the size of the network, it is necessary for the individual index to be standardized between 0.0 (even then it is never happened) and 1.0. It allows, so, meaningful comparisons of performers' closeness centralities across different sizes of workflow-supported org-social networks.

\subsubsection{The Group Closeness Centrality}

As the last step of the analysis phase, there remains one more additional calculation to quantify the network-wide degree of closeness centralization. The network-wide degree of closeness centralization measurement is to quantify the degree of dispersion indicating the hierarchy of closeness centralities within a workflow-supported org-social network. In other words, this measure implies the extent to which performers in a given network differ in their closeness centralities, and it can be calculated by the formula (4) of the index of group closeness centrality.

- The Index of Group Closeness Centrality

$$
G C c=\frac{\sum_{i=1}^{N}\left[C_{C}^{S}\left(\phi^{*}\right)-C_{C}^{S}\left(\varnothing_{i}\right)\right]}{\frac{(N-2)(N-1)}{(2 N-3)}}
$$

In the formula (4), $C_{C}^{S}\left(\phi^{*}\right)$ denotes the highest standardized individual closeness centrality measure observed in a given network, and $C_{C}^{S}\left(\emptyset_{i}\right)$ is the standardized individual closeness centrality measure of each of the $(N-1)$ other performers. The maximum value of the index of group closeness centrality ought to be 1.0 when the corresponding network forms completely an uneven dispersion in the standardized individual closeness centrality measures, which is in the case of that a single performer has the maximum measure and all others have the minimum. In contrast, the index of group closeness centrality equals to 0.0 in the case of that every performer has the same individual closeness centrality measure. Conclusively, the index of group closeness centrality in a workflow-supported org-social network ought to be between 0.0 and 1.0. The closer that the index value is to 1.0, the more uneven or hierarchical is the closeness centralizations of performers in a given network; while on the other hand, the closer 
the index value is to 0.0 , then the more the closeness centralization of the network is evenly dispersed.

\subsection{An Extension of the WSSN-GraphML Schema for Closeness Centralities}

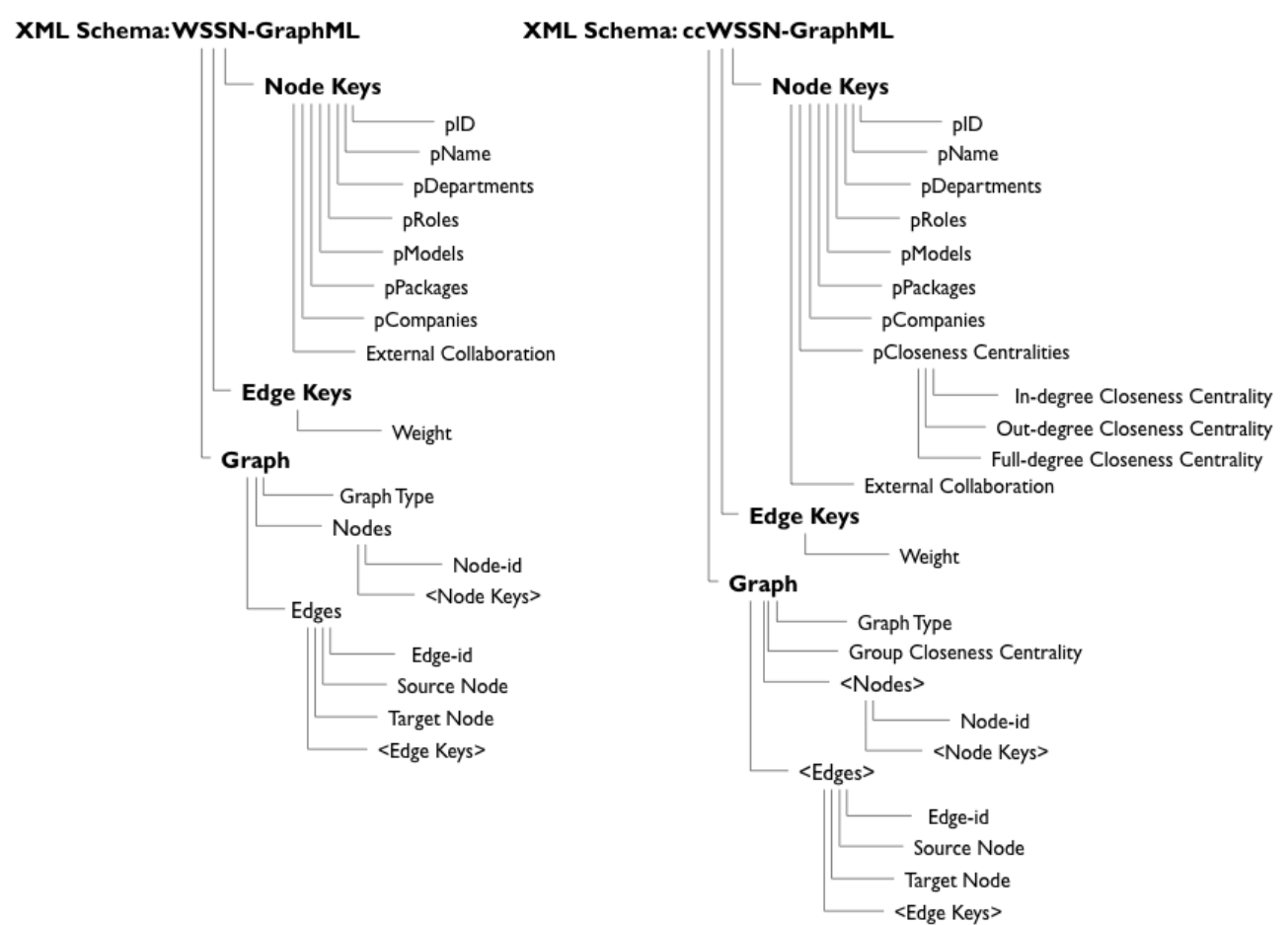

Fig. 1. WSSN's Extended GraphML Schema and ccGraphML Schema for Closeness Centrality

As stated in the introductory statements of the framework, we are particularly interested in visualizing the degree of work-intimacy and collaboration (closeness centrality) of every workflow performer associated with a specific workflow-supported org-social network. At this moment, we would emphasize that, as a future work of the paper, we have a plan to extensively apply the visualization framework to not only a group of workflow models but also all the organization-wide workflow packages, and then definitely the visualization functionality will be the most crucial in the framework and its implemented system ${ }^{5}$.

In order to visualize the quantities of the closeness centrality vector, we need to extend the WSSN-GraphML schema, and devise a new schema of ccGraphML for representing the closeness centrality vector, $\left[\left(C_{C}\left(\varnothing_{1}\right), \ldots, C_{C}\left(\varnothing_{n}\right)\right]\right.$. As depicted in Fig. 4, the XML schema of the proposed ccGraphML basically expresses the graphical magnitude and color of the individual closeness centrality as well as three types (in-degree, out-degree, and full-degree) of closeness centralities on each workflow-performer. In order to sophisticatedly describe the workflow-supported organizational social network by using WSSN-GraphML, ccGraphML supplemented the domain-specific attributes to <Node Keys> and <Edge Keys> as shown in the right-hand side of Fig. 4. Especially, to graphically reflect the strengths of the relationships

\footnotetext{
${ }^{5}$ The authors' research group has been collaboratively conducting a on-going research and development project implementing a Prefuse-based information visualization system as a workflow-supported organizational intelligence solution-suite.
} 
(or work-intimacies) between workflow performers, we adopt the attribute of Weight as <Edge Keys>.

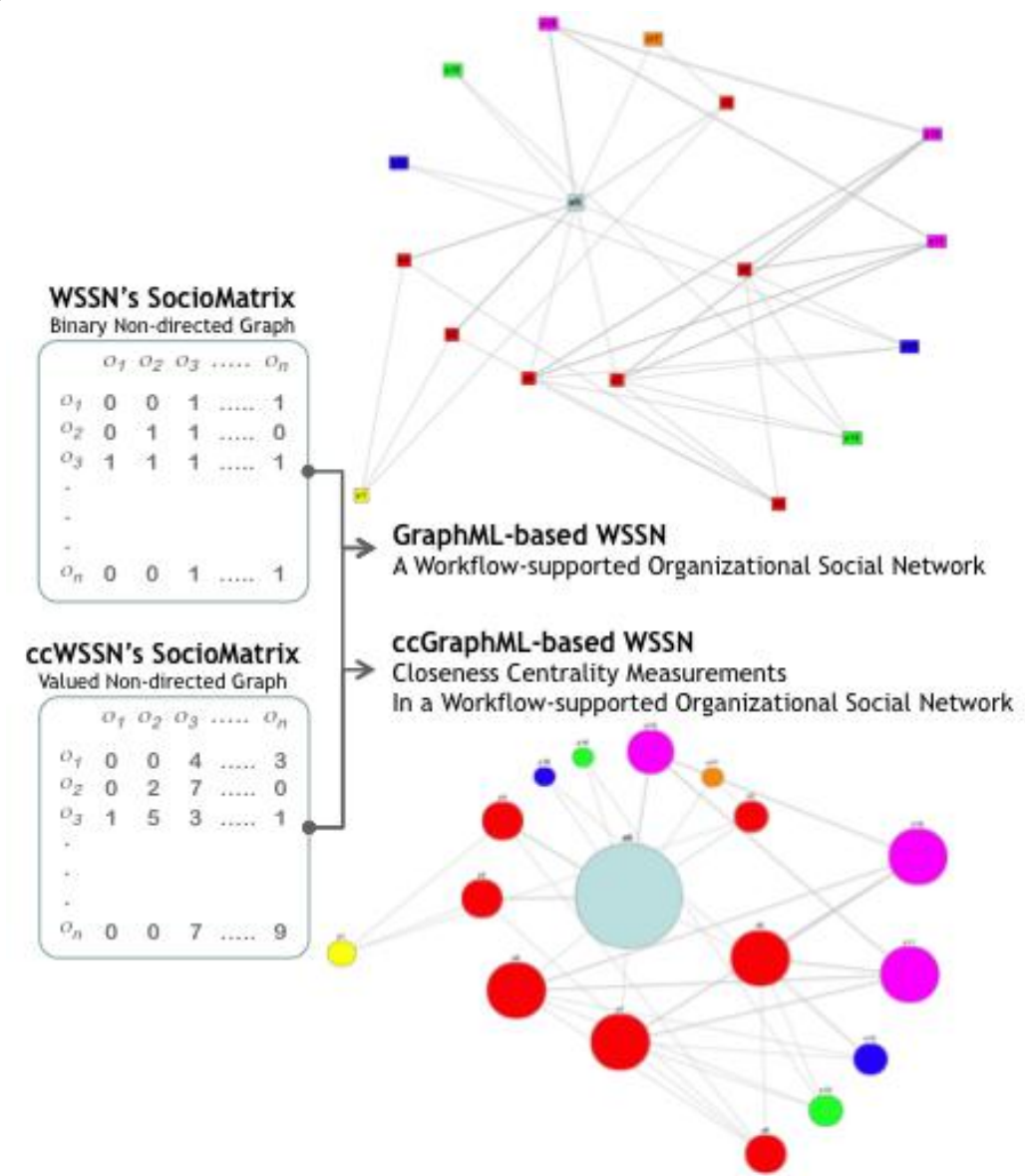

Fig. 2. Closeness Centrality Visualization with ccGraphML for the Performer, $\varnothing_{5}$

Fig. 5 is the ccGraphML contents of the performer-flow graph spawned from the hiring workflow procedure [2] and a screen-snapshot ${ }^{6}$ of the visual representation of the individual closeness centrality measures centered from the performer, $\varnothing_{5}$, who has the highest closeness centrality measure. As you can easily recognize on the colored screen-snapshot of the closeness centralization graph, the centered node $\left(\emptyset_{5}\right)$ is the biggest sized circle, the direct-tied (its geodesic distance is 1.) nodes with $\emptyset_{5}$ are $\emptyset_{2}, \emptyset_{3}, \emptyset_{4}, \emptyset_{5}, \emptyset_{7}, \emptyset_{8}, \varnothing_{9}, \emptyset_{14}, \emptyset_{15}, \emptyset_{16}$, and $\emptyset_{17}$, and the nodes away from $\emptyset_{5}$ as much as 2-tie (its geodesic distance is 2.) are $\emptyset_{1}, \emptyset_{6}, \emptyset_{10}, \emptyset_{11}, \emptyset_{12}$, and $\emptyset_{13}$. Also, it is visually noticeable that the sizes of nodes are figured differently according to the magnitude of the individual closeness centrality index.

Conclusively, the ccGraphML schema can be used for graphically visualizing the individual closeness centralities of performers as well as the group closeness centralities of workflow-supported organizational social networks. The rationale of the ccGraphML schema, so, is on the fact that it pursuits the independency between the closeness centrality measuring component and the closeness centrality visualizing component in implementing a workflow-supported intelligent system based on the theoretical framework of this paper. In other words, it is possible for the visualizing component to visualize a variety of graphical

\footnotetext{
${ }^{6}$ This screen-snapshot is captured from the system's closeness centralization measurement module.
} 
shapes and colors in representing the closeness centralities of workflow performers without the interferences or revisions of the internal measuring component of the system, and vice versa.

\subsection{Key Contributions: Closeness Centralization Visualization in a Workflow-Supported Organization}

The authors' research group has sought the most reasonable metric units for evaluating the degrees of collaborations among people in the workflow-supported people system, and we have found one of them out at last, which is Centrality ${ }^{7}$ [5] stemmed from the social network literature. The most widely used centrality measures are degree, closeness, betweenness, and eigenvalue. In this paper, we are particularly interested in adopting the concept of closeness centrality to measure the degree of work-intimacy and collaboration among performers in enacting a corresponding workflow model. The concept was originally developed to reflect how near a node is to the other nodes in a social network, and so the semantic significance of closeness and distance refer to how quickly an actor can interact with others. In the domain of workflow-supported organizational knowledge, the performer's closeness centralization concept can be interpreted as the extent to how much close a specific performer is to the others on a flow of works. Fig. 1 shows the conceptual significance of the performer's closeness centralization. The activity-performer associations eventually form a flow of works among the workflow-performers, which can be represented by the actor-based workflow model [1] or the workflow-supported organizational social network model [13], both of which were proposed by the authors' research group.

Then, on a workflow-supported organizational social network, it might be quite in the nature of things to raise the questions as followings:

- Who is the most important or prominent performer(s) interacting the most tightly with others in enacting a specific workflow procedure?

- How to visualize the degree of the most prominent performer's closeness centrality to others in a workflow-supported organizational social network?

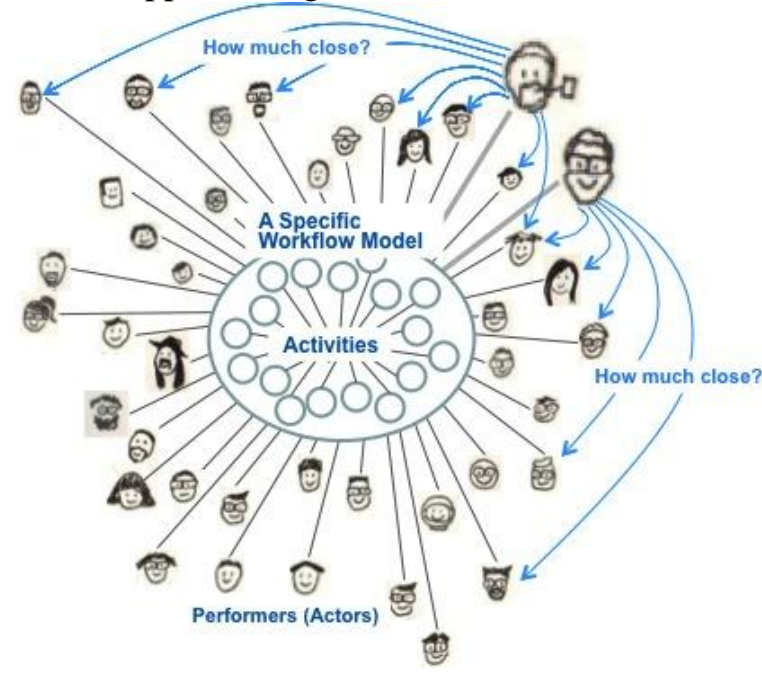

Fig. 3. Performers' Involvement and Closenesses on a Specific Workflow Model

\footnotetext{
${ }^{7}$ Centrality, where a prominent actor has high involvement in many relations, regardless of whether sending and receiving ties, in a social network.
} 
Conclusively, the answers to the questions ought to be able to convey a very valuable and meaningful insight to the workflow-supported organization. Specially, the visualization framework in this paper implies not only for visualizing a shape of workflow-performers' collaborative relationships through a workflow-supported organizational social network but also for quantifying their closeness centrality measures by mathematically extending some of the well-known closeness centrality formulas [5] in the social network analysis literature. Ultimately, the visualization framework will be a graphical basis for implementing an organizational intelligent system to quantitatively and graphicaly answer to the questions through the closeness centralization concept and measurement - closeness-centrality - at both individual and group levels of the workflow-supported organization.

\section{Conclusion}

In this paper, we have proposed a means of visualizing the closeness centrality measures that are quantitatively expressing the prominency and collaborative behaviors among workflow-supported performers in enacting a workflow procedure. Through the visualization framework, so, we can straightforwardly implement an automatic visualization system for the closeness centrality measurements as well as for workflow-supported organizational social networking knowledge management. Likewise, as future works, we need not only to elaborate on the functional expansion of the closeness centrality formulas and the WSSN-GraphML and WSSN-ccGraphML schemas so as for the framework to handle a group of workflow procedures (or a workflow package) with organization-wide workflow-performers, but also to develop the remainder centrality analysis techniques, like betweenness and eigenvalue centralities, to be applied to workflow-supported organizational social networks. The issue of scalability as well as extendibility is in our sights as well, as the same measurements and effects must be achieved in larger workflow-performers and groups.

\section{Acknowledgements}

This research was supported by the MSIP (Ministry of Science, ICT and Future Planning), Korea, under the "IT Consilience Creative Program" (NIPA-2014-H0201-14-1002) supervised by the NIPA (National IT Industry Promotion Agency). Additionally, the authors would like to give special thanks to our colleagues and the institutes, the School of Integrated Technology at YONSEI UNIVERSITY, the collaboration technology research lab at KYONGGI UNIVERSITY, and the R\&D Center at BISTel, Inc., for supporting this collaborative research. Also, this research was partially supported by the GRRC contents convergence software research center at Gyeonggi Province.

\section{References}

[1] Kim, K., et al., “Actor-oriented Workflow Model," in Proc. of the 2nd international symposium on Cooperative Database Systems for Advanced Applications, Wollongong, Australia, March 27-28, pp. 163-177, 1999 Article (CrossRef Link)

[2] Kim, K., Ellis, C. A., "Section II / Chapter VII. An ICN-based Workflow Model and Its Advances," Handbook of Research on BP Modeling, pp. 142-172, IGI Global, ISR, pp. 142-172, 2009 Article (CrossRef Link)

[3] Won, J., "A Framework: Organizational Network Discovery on Workflows," Ph.D. Dissertation, 
Department of Computer Science, KYONGGI UNIVERSITY, 2008 Article (CrossRef Link)

[4] Skerlavaj, M., Dimovski, V., Desouza, K. C., "Patterns and structures of intra-organizational learning networks within a knowledge-intensive organization," Journal of Information Technology, vol. 25, no. 2, pp. 189-204, 2010

[5] Knoke, D., Yang, S., "SOCIAL NETWORK ANALYSIS - 2nd Edition, Series: Quantitative Applications in the Social Sciences," SAGE Publications, 2008 Article (CrossRef Link)

[6] Poelmans, S., Reijers, H. A., Recker, J., "Investigating the Success of Operational Business Process Management Systems," Information Technology and Management, vol. 14, Iss. 4, pp. 295-314, 2013

[7] Aalst, W. M. P., et al., "Discovering Social Networks from Event Logs," COMPUTER SUPPORTED COOPERATIVE WORK, vol. 14, No. 6, pp. 549-593, 2005 Article (CrossRef Link)

[8] Oinas-Kukkonen, H., et al., "Social Networks and Information Systems: Ongoing and Future Research Streams," JOURNAL OF THE ASSOCIATION OF INFORMATION SYSTEMS, vol. 11, Iss. 2, pp. 61-68, 2010 Article (CrossRef Link)

[9] Song, J., et al., "A Framework: Workflow-based Social Network Discovery and Analysis," in Proc. of the International Workshop on Workflow Management in Service and Cloud Computing, Hongkong, China, pp. 421-426, 2010

[10] Kim, K., "A Workflow-based Social Network Discovery and Analysis System," in Proc. of the International Symposium on Data-driven Process Discovery and Analysis, Campione d'Italia, Italy, June 29-July 1, pp. 163-176, 2011 Article (CrossRef Link)

[11] Kim, H., et al., "A Workflow Affiliation Network Discovery Algorithm," ICIC Express Letters, vol. 6, No. 3, pp. 765-770, 2011

[12] Park, S., et al., "A Closeness Centrality Analysis Algorithm for Workflow-supported Social Networks," in Proc. of IEEE International Conference on Advanced Communications Technology, Pheonix Park, Pyeongchang, South Korea, pp. 158-161, 2013

[13] Jeon, M., et al., "A Workflow-supported Social Network Model," in Proc. of the ACIS/JNU International Conference on Computer, Networks, Systems, and Industrial Engineering, Jeju Island, South Korea, May 23-25, pp. 457-461, 2012

[14] Ra, I., et al., "ccWSSN-GraphML: An Extended Graph Markup Language for Visualizing Closeness-centrality Measurements of Workflow-supported Org-social Networks," in Proc. of KSII the 8th Asia Pacific International Conference on Information Science and Technology (APIC-IST), Jeju Island, South Korea, pp. 97-100, 2013

[15] Kaufman, J. H., Edlund, S., Ford, D. A., Powers, C., "The Social Contract Core," ELECTRONIC COMMERCE RESEARCH, vol. 5, Iss. 1, pp. 141-165, 2005

[16] Chuang, S., Liao, C., Lin, S., "Determinants of Knowledge Management with Information Technology Support Impact on Firm Performance," Information Technology and Management, vol. 14, Iss. 3, pp. 217-230, 2013

[17] Aier, S., "The Role of Organizational Culture for Grounding, Management, Guidance and Effectiveness of Enterprise Architecture Principles," Information Systems and e-Business Management, vol. 12, Iss. 1, pp. 43-70, 2014

[18] Ferneley, E., Helms, R., "Editorial of the Special Issue on Social Networking," Journal of Information Technology, vol. 25, No. 2, pp. 107-108, 2010

[19] Faust, K., "Centrality in Affiliation Networks," Journal of Social Networks, vol. 19, pp. 157-191, 1997 Article (CrossRef Link)

[20] Battsetseg, A., et al., "Organizational Closeness Centrality Analysis on Workflow-supported Activity-Performer Affiliation Networks," in Proc. of IEEE International Conference on Advanced Communications Technology, Pheonix Park, Pyeongchang, South Korea, pp. 154-157, 2013

[21] Jeong, H., et al., "Betweenness Centralization Analysis Formalisms on Workflow-Supported Org-Social Networks," in Proc. of IEEE International Conference on Advanced Communications Technology, Pheonix Park, Pyeongchang, South Korea, 2014

[22] Jeong, H., et al., "A Workflow-supported Social Networking Knowledge Visualization System," in Proc. of the 5th International Conference on Internet, Pattaya, Thailand, pp.187-193, 2013 
[23] Komlodi, A., et al., “An Information Visualization Framework for Intrusion Detection,” in Proc. of ACM International Conference on CHI, Vienna, Austria, pp. 1743-1746, 2004

[24] Heer, J., Card, S. K., Landay, J. A., "prefuse: A Toolkit for Interactive Information Visualization," in Proc. of ACM International Conference on CHI, Portland, Oregon, USA, 2005

[25] Meyer, M., Girba, T., Lungu, M., "Mondrian: An Agile Information Visualization Framework," in Proc. of ACM International Conference on SOFTVIS, Brighton, United Kingdom, pp. 135-144, 2006
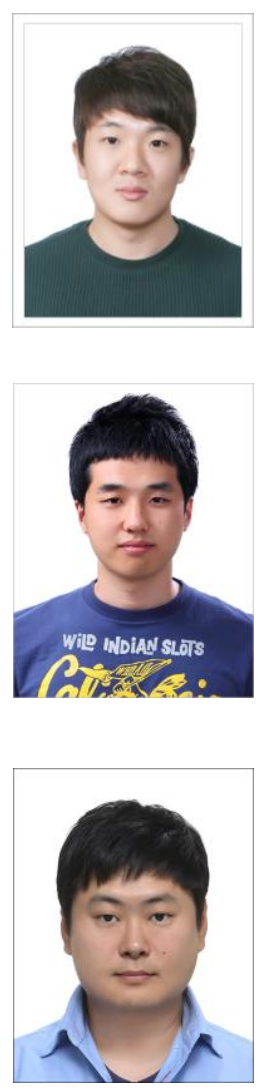

Min-Joon Kim is a full-time master's student at the School of Integrated Technology at Yonsei University. He received his B.S. in computer science from the University of Colorado at Boulder in 2011. His research interests include intelligent database systems, meta-data analysis, Internet of Things (IoT), computer vision, and image processing.

Hyun Ahn is a full-time Ph. D. student of computer science department and a graduate member of the collaboration technology research laboratory at Kyonggi University, South Korea. He received B.S. and M.S. degrees in computer science from Kyonggi University in 2011 and 2013, respectively. His research interests include workflow systems, BPM, scientific workflow systems, workflow-supported social and affiliation networks discovery, analysis, and visualization.

Minjae Park is a senior member of research staff at the solution R\&D research center of BISTel, Inc., South Korea. He received B.S., M.S., and Ph.D. degrees in computer science from Kyonggi University in 2004, 2006, and 2009, respectively. His research interests include groupware, workflow systems, BPM, CSCW, collaboration theory, process warehousing and mining, workflow-supported social networks discovery and analysis, and process-aware factory automation systems. 